(C) He G., Zhou X., Shi Y., Wang D., Yu J., Xing H., Yin H., Zhang J., Liu H., Wang B., Jia Y., Zhang Y., 2021

DOI 10.18019/1028-4427-2021-27-3-374-382

\title{
The monitoring value of ultrasonography in the treatment of bone union at the docking site after tibial bone transport by accordion maneuver
}

\author{
G. He, X. Zhou, Y. Shi, D. Wang, J. Yu, H. Xing, H. Yin, J. Zhang, H. Liu, B. Wang, Y. Jia, Y. Zhang \\ The second Hospital of Shanxi Medical University, Taiyuan, China
}

\begin{abstract}
Purpose To explore the value of ultrasound in the treatment of bone union at the docking site by accordion technique. Methods Sixteen patients were recruited. The accordion operation was performed at the docking site after bone transport. The specific operation process was compression, suspension of adjustment, distraction, retraction. Ultrasound was used to monitor the hematoma, blood flow and callus in and around the docking site at each stage. Results (1) At the compression, there were 14 cases with hematoma and 2 cases without hematoma. (2) The size of hematoma was negatively correlated with the bone healing time of the docking site and the blood flow resistance index. (3) The Adler grading of blood flow gradually increased before the retraction, and then gradually decreased. But the degree of callus mineralization continued to rise. Conclusion (1) Ultrasound can dynamically monitor the change trend of hematoma, blood flow and new callus at the docking site during accordion operation, so as to predict the tendency of bone healing at the docking site. (2) This study can preliminarily demonstrate that the compression stress applied in accordion technique can promote the bone healing at the docking site.

Keywords: ultrasonography; accordion maneuver; hematoma; blood flow; bone callus
\end{abstract}

\section{INTRODUCTION}

Ilizarov technique, also known as bone transport technique, can provide continuous, stable and slow distraction stimulation to living bone tissue after osteotomy $[1,2]$. Under the stimulation of this stress, the regeneration signal system of living bone tissue is activated and amplified, so that it can grow beyond the normal rate of osteogenesis, and the soft tissue around it, including nerves, muscles, blood vessels, skin and so on, can also regenerate synchronously. This technique has been widely used in clinical practice and has achieved satisfactory results [3], especially in the treatment of bone defects caused by open comminuted fracture of long bone $[4,5]$ and chronic osteomyelitis [6] and other diseases. However, this technique also has many complications, among which is the difficulty of bone healing at the docking site [7, 8]. At present, the main treatment for this complication is bone cortical exfoliation $[9,10]$ and autologous bone transplantation, but the surgical trauma of this method is large and accompanied by the injury of the donor area. Accordion maneuver is a minimally invasive osteogenesis technology derived from the principle of bone transport technology, which induces bone regeneration at the docking site through axial compression, distraction and retraction.
At present, there are many clinical methods to monitor callus formation during bone healing, and X-ray is one of the common methods. It plays a significant role in the process of callus monitoring in the late stage of bone healing, while the operation of accordion technology belongs to the early stage of bone healing, the amount of callus is small, and the X-ray imaging is not obvious. Therefore, during the operation of the accordion, X-ray is not an ideal means of monitoring.

Inrecentyears, withthedevelopmentofmusculoskeletal ultrasound, ultrasound has been gradually applied in the diagnosis and treatment of orthopedic diseases [11-13], among which high-frequency color doppler ultrasound is gradually applied to monitor the formation process of callus in the early stage of bone healing. At present, it has been reported that application of ultrasound can observe the early signs of bone healing after fracture $[14,15]$, and evaluate the formation of early new callus during bone transport $[16,17]$. However, there is no report on the application of ultrasound to monitor the bone healing at the docking site. Therefore, the purpose of this paper is to use ultrasound to monitor the early changes of the union of bone at the docking site during the operation of accordion, and to further explore the value of ultrasonic monitoring.

\section{PATIENTS AND METHODS}

\section{Patients}

From July 2018 to August 2020, 16 patients with tibial bone transport were consecutively collected from the same hospital, including 14 males and 2 females, aged 6-61 years, with an average age of $45.25 \pm 14.3$ years. Before bone transport, the tibial bone defect ranged from 2.04 to $12.07 \mathrm{~cm}$, with an average of $5.99 \pm 2.6 \mathrm{~cm}$ (Table 1). The bone defects in 16 patients were caused by one open fracture with bone defect, 14 chronic osteomyelitis, and one fracture with tibiofibular deformity. One patient had type 2 diabetes and three had equinus deformity. Inclusion criteria: (1) patients with contact at the docking site after tibial bone transport; (2) patients who perform accordion operation at the docking site; (3) the infection test is normal. Exclusion criteria: (1) Adverse skin conditions, such as unhealed skin and severe depressions on the skin surface; (2) Poor compliance, unable to cooperate with the return visit on

[ᄄ] He G., Zhou X., Shi Y., Wang D., Yu J., Xing H., Yin H., Zhang J., Liu H., Wang B., Jia Y., Zhang Y. The monitoring value of ultrasonography in the treatment of bone union at the docking site after tibial bone transport by accordion maneuver. Genij Ortopedii, 2021, vol. 27, no 3, pp. 374-382. DOI 10.18019/1028-4427-2021-27-3-374-382 
time; (3) The ultrasonic probe could not be placed due to the obstruction of the external frame.

\section{Surgical methods}

All patients were operated by the same experienced clinician, and the operation was performed in strict accordance with Ilizarov technique. Lumbar combined anesthesia or spinal anesthesia was used. The affected limb was routinely disinfected and sterile sheet was laid. Annular external fixator for bone transport was placed in the affected limbs (Beijing ruilang medical equipment co., LTD.). The external fixator of the lower leg was a five-ring configuration, with two rings at the distal and proximal ends and one ring in the middle. If the affected limbs were combined with equinus deformity, equinus orthopedic configuration should be added. Three Kirschner wires with a diameter of $2.0 \mathrm{~mm}$ were intercrossed in the two rings at the proximal ends of the tibia, one of which penetrates the tibiofibula. The distal wires were threaded in a similar manner to the proximal. In the case of bone defect in the middle and lower tibia, incision about $1 \mathrm{~cm}$ long was made under the tibial tubercle; in the case of bone defect in the upper tibia, incision was made in the middle of the tibia, and a minimally invasive, closed and transverse osteotomy was performed, followed by a closed incision. On both sides of the osteotomy end, three threaded half needles with a diameter of 4.0 or $5.0 \mathrm{~mm}$ were inserted in different directions to fix and distract the conveying bone segment, and enhance the stiffness of the external fixator to reduce elastic deformation. After that, the infected and necrotic bone and soft tissue in the wound were thoroughly excised, and the wound was washed successively with hydrogen peroxide and flushing saline. The bone defect area was filled with antibiotic bone cement beads. It was prepared by mixing $20 \mathrm{~g}$ PALACOS ${ }^{\circledR} \mathrm{R}+\mathrm{G}$ bone cement powder(including $0.25 \mathrm{~g}$ of gendamycin, Heraeus Medical GmbH, Wehrhe IM, Germany) with $3 \mathrm{~g}$ of sensitive antibiotic, adding the liquid part of bone cement, mixing well, and making soya bean to peanut size bead connected with 2 ligament wire before solidifying.

Postoperative treatment

The sliding segment was slowly distracted through the external fixator at 12 days after the operation. The adjustment method is to adjust the adjustment nut on the threaded connection rod of the annular external fixator. The adjusting nut was composed of six sides, and the axial movement distance was one millimeter for each rotation circle. The adjustment rate was one side at a time and five times a day, that is, the adjustment rate was 0.83 millimeter per day. At the same time, the neeedles should be disinfected 2-3 times a day with alcohol swab to prevent infection. As the bone segments were transported, the antibiotic bone cement beads at the bone defect were gradually pulled out, and the space at the bone defect became smaller and smaller until the bone was completely contacted and the docking sites were formed.

The accordion operation would be started after the contact at the docking site. The specific operation process was compression for two weeks, suspension of adjustment for 12 days, distraction for two weeks, retraction for two weeks. The adjustment method is to adjust the adjustment nut on the threaded connection rod of the annular external fixator. The adjusting nut was composed of six sides, and the axial movement distance was one millimeter for each rotation circle. The adjustment rate was one side at a time and five times a day, that is, the adjustment rate was 0.83 millimeter per day. Ultrasound (The experimental instrument was GE LOGIQ E9 ultrasonic instrument, and the frequency of ultrasonic probe was $6-15 \mathrm{MHz}$ ) was used to observe the bone healing at the docking site during contact, at each stage of accordion operation and at the stage of consolidation and mineralization. In order to improve the accuracy of the data, the ultrasound examination was carried out by senior ultrasound physician who was specially responsible for musculoskeletal ultrasound. To reduce ultrasonic attenuation, the ultrasound probe should be placed at the thin soft tissue layer at the docking site, such as the anterior tibia and/or the medial side. In order to obtain transverse and longitudinal scanning audiovisual images on the docking site, the probe should be placed in a direction parallel and vertical to the longitudinal axis of the tibia [14]. Before and after the accordion operation, X-ray was applied to observe the osteogenesis on the docking site (The X-ray came from Philips Digital Diagnost DR3.1).

Table 1

The table shows the basic information of 16 patients

\begin{tabular}{|c|c|c|c|c|c|}
\hline Case number & Age & Sex & Cause of disease & Bone defect range/cm & The site of a bone defect in the tibia \\
\hline 1 & 42 & F & Chronic osteomyelitis & 3.38 & Middle and lower (R) \\
\hline 2 & 60 & M & Chronic osteomyelitis & 3.09 & Middle and lower (L) \\
\hline 3 & 44 & M & Chronic osteomyelitis & 5.30 & Middle and lower (L) \\
\hline 4 & 41 & M & Open fracture & 2.66 & Distal end (L) \\
\hline 5 & 55 & M & Chronic osteomyelitis & 7.66 & Midtal end (L) \\
\hline 6 & 37 & M & Chronic osteomyelitis & 5.27 & Middle (L) (R) \\
\hline 7 & 61 & M & Chronic osteomyelitis & 8.71 & Middle and lower (R) \\
\hline 8 & 56 & M & Chronic osteomyelitis & 5.78 & Middle (L) \\
\hline 9 & 43 & M & Chronic osteomyelitis & 5.43 & Distal end (R) \\
\hline 10 & 54 & M & Chronic osteomyelitis & 5.97 & Distal end (L) \\
\hline 11 & 56 & M & Chronic osteomyelitis & 7.44 & Proximal end (R) \\
\hline 12 & 41 & M & Chronic osteomyelitis & 12.07 & Middle (L) \\
\hline 13 & 33 & M & Chronic osteomyelitis & 6.01 & Middle (R) \\
\hline 14 & 61 & M & Chronic osteomyelitis & 9.03 & 6.02 \\
\hline 15 & 34 & M & Chronic osteomyelitis & 2.04 & \\
\hline 16 & 6 & F & Fracture with bone deformity & & \\
\hline
\end{tabular}


The observation indices under ultrasound were the hematoma on the lateral side of the cortical bone, the blood flow signal inside and around, the shape and density of new formed callus at the docking site. (1) Observe whether there was hematoma on the lateral side of the cortical bone at the docking site When compressed for 2 weeks. if there was hematoma, measure its range, the unit was square centimeter. (2) During each stage of accordion operation, observe the blood flow signal in and around the docking site, measure the blood flow parameters at each point in the target area, such as peak systolic velocity (Vs), lowest diastolic velocity (Vd), and RI, and calculate the RI mean. The abundance of blood flow signal was observed and classified by Adler blood flow semi-quantitative grading [18]. Grade 0: no blood flow signal could be detected within the target area (within the diameter range of $1-2 \mathrm{~cm}$ at the docking site). Grade 0: no blood flow signal could be detected within the target area (within the diameter range of $1 \sim 2 \mathrm{~cm}$ at the docking site). Grade I: a small amount of blood flow signal could be detected in the target area, and spot-like blood flow signal could be seen at one or two places. The inner diameter of blood vessels was less than $1 \mathrm{~mm}$. Grade II: Moderate blood flow signal could be detected in the target area, including three small blood vessels or one long blood vessel. Grade III: abundant blood flow signal could be seen in the target area, seen in more than four at the same time, some blood vessels were interwoven connected net-like. (3) Observe the shape and density of new callus at each stage of accordion operation and the bone connection at the docking site. According to the shape and density of callus, it could be divided into no callus, fibrous callus, isolated spot-like bone callus, fused flaky bone callus and linear bone callus [19].

At the last follow-up, bone healing was assessed according to evaluation criteria of Paley for bone defects [20], which could be divided into four grades: excellent, good, acceptable and poor. The excellent criteria were fracture healing, no recurrent infection, residual deformity $<7^{\circ}$, unequal limb length $<2.5 \mathrm{~cm}$. The criterion of good was fracture healing, combined with any two of the other three. The acceptable criterion was fracture healing, and any one of the other three items was combined. Poor criteria for fracture had not yet healed or re-fracture.

\section{Statistical analysis}

SPSS25.0 statistical software was used for statistical analysis. Pearson correlation coefficient was used to analyze the correlation between hematoma range at two weeks of compression and RI mean at two weeks of distraction in 16 patients, and the correlation between the hematoma range at two weeks of compression and the bone healing time at the docking site in 14 patients with hematoma.

\section{RESULTS}

\section{Changes of hematoma under ultrasound}

It was observed that in 14 of the 16 patients, hematoma (no or hypoechoic fluid dark area under ultrasound) appeared on the lateral side of cortical bone on the docking site at two weeks of compression (Fig. 1). The hematoma was measured at the end of compression in 14 patients ranged from 0.103 to $0.644 \mathrm{~cm}^{2}$, with an average of $\left(0.410 \pm 0.170 \mathrm{~cm}^{2}\right)$. The hematoma was gradually organized from the periphery to the center during the period of suspended regulation and distraction (the echo of the hematoma was gradually increased under ultrasound). No hematoma appeared in the other two patients. After cortical stripping was performed at the docking site, and then it was pressurized again, and hematoma was observed on the lateral cortical bone by ultrasound.

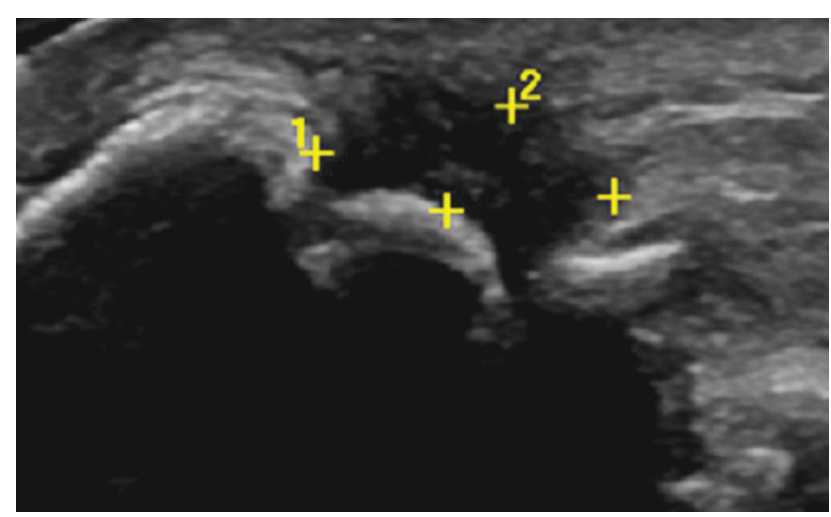

Fig. 1 The picture was the transverse scan sonogram on the docking site of the tibia. In the figure, the anechoic fluid dark area was hematoma
Changes of Adler grade of blood flow and bone callus morphology and density in different stages of accordion operation under ultrasound

In 14 patients with hematoma, when the docking site was in contact, no or a small amount of spot-like blood flow signal was observed, the Adler grade was 0-I, and no or a small amount of hypoechoic fibrous callus was observed (Fig. 2a). A small amount of blood flow signal surrounding the hematoma could be seen during the period of compression and suspended regulation. With the progress of hematoma organization, blood flow gradually extended into the hematoma, and the blood flow signal Adler was graded as I-II. Fibrous callus with low to moderate echo could be seen (Fig. 2b). In the distraction stage, dotted, small linear and clustered blood flow distribution could be seen, and the Adler grade was grade II-III. At this time, the callus also changed from medium echo fibrous callus to high echo punctate bone callus (Fig. 2c). During the retraction period, the blood flow signal decreased, and the Adler grade was III-II. At this time, the callus gradually changed from point shape to small flake shape, and the echo intensity also gradually increased (Fig. 2d). After contacted again, the blood flow signal decreased to level II-0. At this time, the callus continued to mineralize until bone healing. In the two patients without hematoma, the amplitude of blood flow signal change was not obvious in each stage of accordion operation, the Adler grade was $0-$ I, and the morphology and density of callus were also significantly slower than those of patients with hematoma at the same time. The specific blood flow Adler grading and callus grading of 16 patients were shown in Fig. 3, Fig. 4, Table 2 and Table 3. 

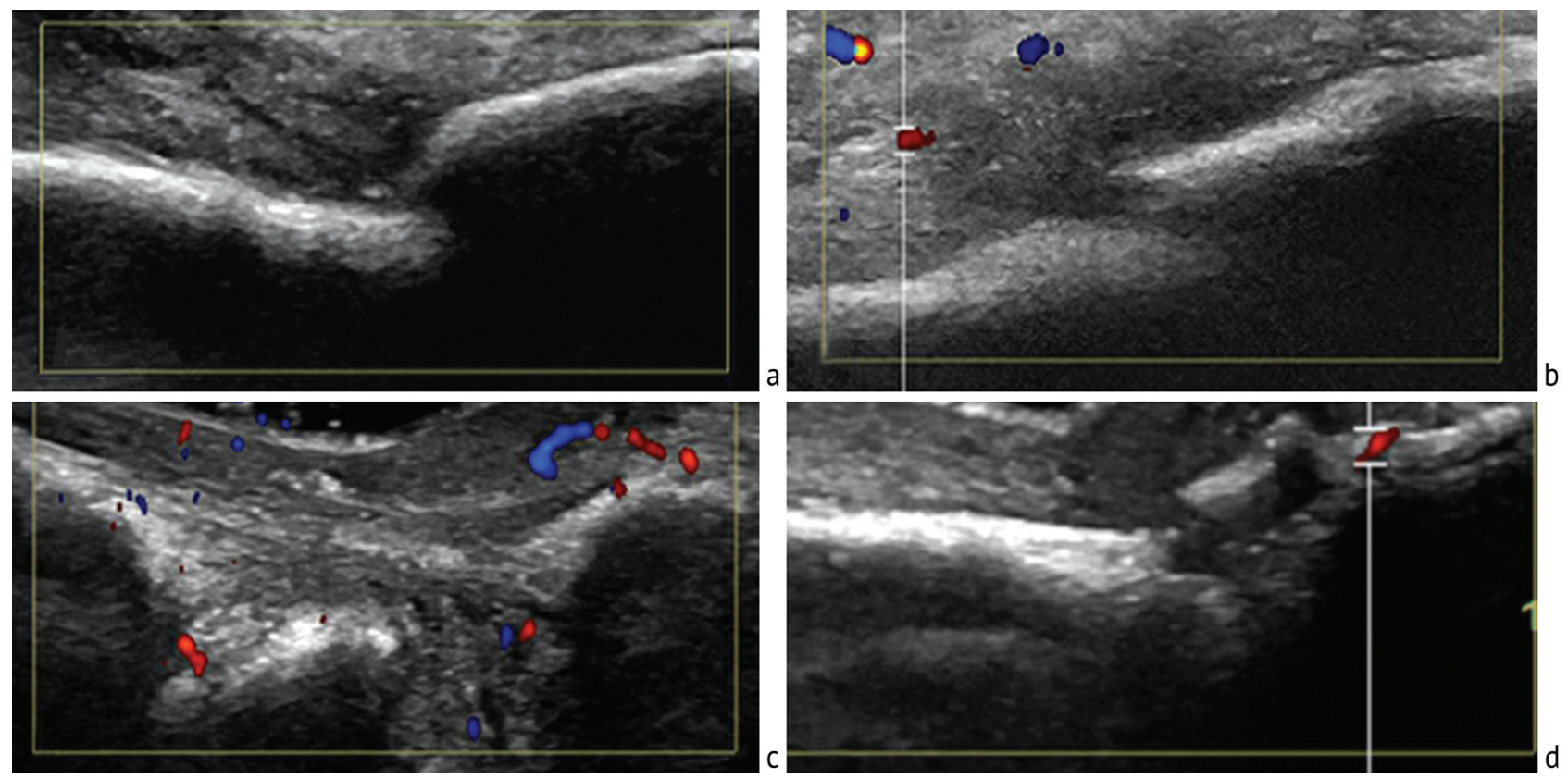

Fig. 2 The Adler grading of the blood flow signal and the shape and density of the callus at the docking site in each stage of accordion operation: $\boldsymbol{a}$ no obvious blood flow signal and new callus when the docking site is in contact; $\boldsymbol{b}$ hematoma, the blood flow signal with Adler grade I, and fibrous callus when pressurized for two weeks; $\boldsymbol{c}$ the blood flow signal with Adler grade III, and the mixed spot-like and flaky bone callus, when distracted for two weeks; $\boldsymbol{d}$ the blood flow signal with Adler grade I, and the flaky bone callus distributed along the cortex when retracted for one weeks

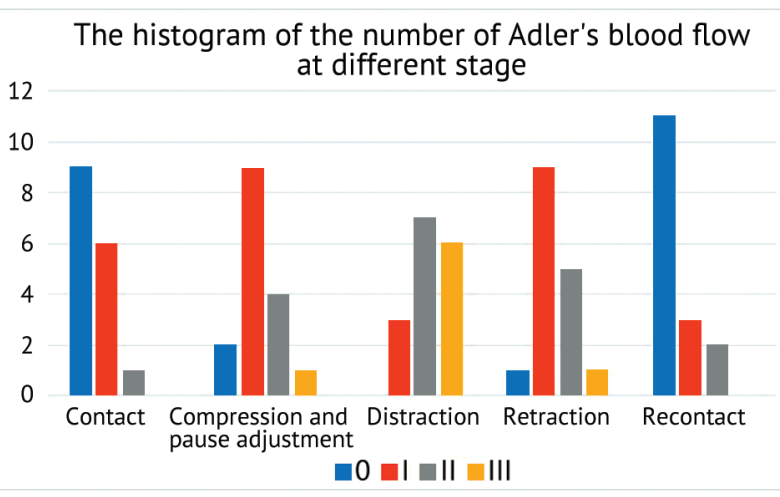

Fig. 3 The histogram of patient frequency distribution of Adler's blood flow at each stage of accordion operation

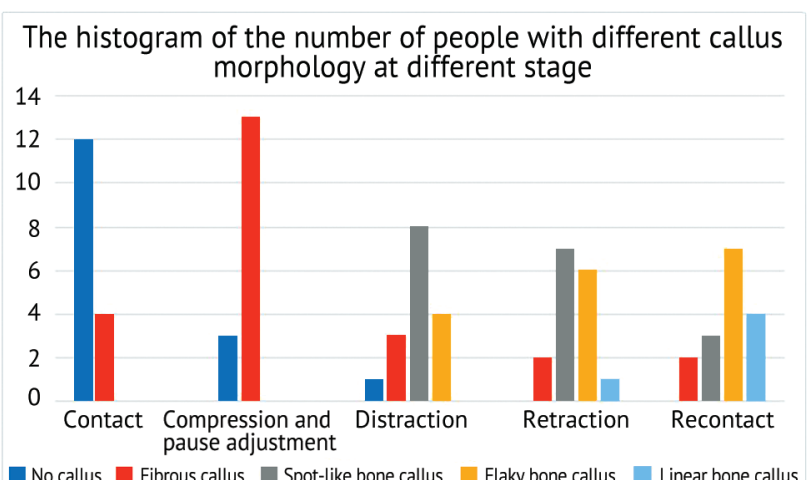

Fig. 4 The histogram of the number of people with different callus morphology at each stage of accordion operation

Table 2

The table shows the distribution of Adler's grading of blood flow at different stages in 16 patients

\begin{tabular}{|l|c|c|c|c|}
\hline & 0 & I & II & III \\
\hline Contact & 9 & 6 & 1 & 0 \\
\hline Compression and pause adjustment & 2 & 9 & 4 & 1 \\
\hline Distraction & 0 & 3 & 7 & 6 \\
\hline Retraction & 1 & 9 & 5 & 1 \\
\hline Re-contact & 11 & 3 & 2 & 0 \\
\hline
\end{tabular}

Table 3

The table shows the distribution of different callus morphology at different stages in 16 patients

\begin{tabular}{|l|c|c|c|c|c|}
\hline & No callus & Fibrous callus & Spot-like bone callus & Flaky bone callus & Linear bone callus \\
\hline Contact & 12 & 4 & 0 & 0 & 0 \\
\hline Compression and pause adjustment & 3 & 13 & 0 & 0 & 0 \\
\hline Distraction & 1 & 3 & 8 & 4 & 0 \\
\hline Retraction & 0 & 2 & 7 & 6 & 1 \\
\hline Re-contact & 0 & 2 & 3 & 7 & 4 \\
\hline
\end{tabular}




\section{The bone healing at the docking site}

In 14 patients with hematoma at the docking site, ultrasonic observation showed that the bone callus continued to mineralize and gradually shaped, and the new formed callus gradually connected the two sides of the docking site, at where the fracture line gradually blurred and disappeared, at this time, ultrasound could no longer penetrate the bone cortex (Fig. 5). X-ray observation showed that the healing time of the docking site ranged from 23 to 40 weeks, with an average of $30.8 \pm 4.9$ weeks (Fig. 6). In the two patients without hematoma, the callus at the docking site grew slowly. After 20 weeks of continuous observation, there was still no obvious signs of union. In order to prevent the occurrence of bone nonunion, bone cortical exfoliation was performed instead. After the operation, ultrasound detected the formation of hematoma at the docking site, and the accordion operation was continued. Finally, bone healing was performed at 30 and 32 weeks after the operation. At the last follow-up, according to the Paley evaluation criteria, the results of bone healing were excellent in 12 cases and good in four cases in this group.

\section{Complications}

In the course of treatment, 5 patients developed needle infection, which gradually subsided after Needle care. The malalignment of the docking site occurred in 2 cases, which was gradually corrected by adjusting the external fixator. None of the 16 patients had allergic reaction or immune rejection.

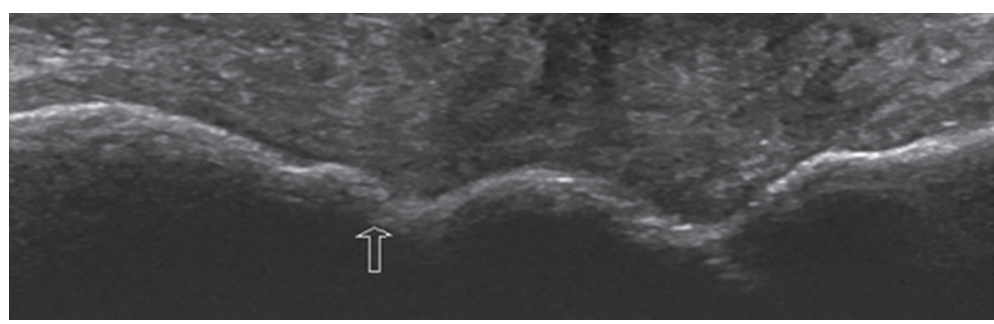

Fig. 5 The image shows a longitudinal scan at the docking site at 25 weeks. The arrow points to the docking site of the tibia, which has been bone connected under ultrasound, and ultrasound wave could no longer penetrate the bone cortex
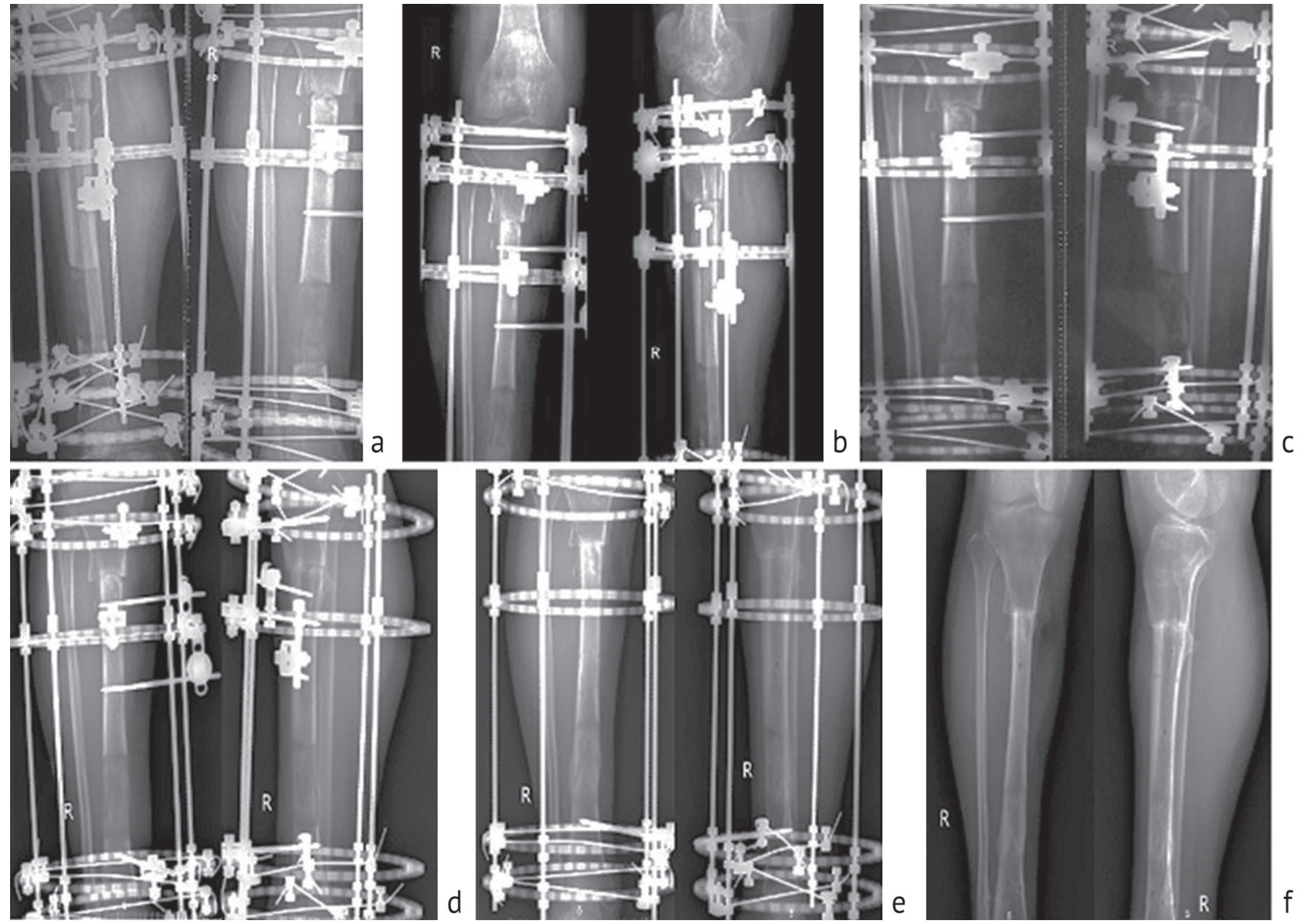

Fig. 6 Shows the X-ray of callus growth at the docking site of the right tibia at different stages before and after accordion operation. The distal tibia was the distraction osteogenic area, and the proximal end was the docking site: $\boldsymbol{a}$ no callus formed before accordion operation; $\boldsymbol{b}$ new callus could be seen after accordion operation; $\boldsymbol{c}$ after four weeks of accordion operation, the callus of new bone was more than before; $\boldsymbol{d}$ obvious callus after eight weeks of accordion operation; $\boldsymbol{e}$ good bone union at the docking site after 22 weeks of accordion operation; $\boldsymbol{f}$ after one year of accordion operation, the external fixator had been removed 


\section{Changes of each index under ultrasound}

The relationship between the hematoma size at two weeks of compression and the RI average at two weeks of retraction in 16 patients was that the greater the hematoma was, the lower the RI average was. Pearson correlation coefficient was -0.801 , showing a linear negative correlation $(\mathrm{P}<0.001)$. In the 14 patients with hematoma, the relationship between the size of the hematoma and the bone healing time of the docking site at two weeks of compression was that the bigger the hematoma was, the shorter the bone healing time was. Pearson correlation coefficient was -0.801 , showing a linear negative correlation $(\mathrm{P}<0.001)$. The specific data was described in Table 4. During accordion operation, Adler grading of blood flow in the docking site of 16 patients increased gradually before retraction, and decreased gradually after retraction. Callus mineralization increased gradually in 16 patients. Specific trend was described in Fig. 7 and Fig. 8.

Table 4

Distribution of hematoma size, mean RI and bone healing time in 16 patients

\begin{tabular}{|c|c|c|c|c|}
\hline NO & $\begin{array}{c}\text { Hematoma size at } 2 \text { weeks } \\
\text { of compression } / \mathrm{cm}^{2}\end{array}$ & $\begin{array}{l}\text { RI mean at } 2 \text { weeks } \\
\text { of distraction }\end{array}$ & Bone healing time /weeks & $\begin{array}{l}\text { Evaluation criteria of Paley } \\
\text { for bone defects }\end{array}$ \\
\hline 1 & 0.604 & 0.47 & 25 & Excellent \\
\hline 2 & 0.516 & 0.38 & 30 & Good (residual deformity $10^{\circ}$ ) \\
\hline 3 & 0.644 & 0.44 & 25 & Excellent \\
\hline 4 & 0.370 & 0.53 & 37 & Excellent \\
\hline 5 & 0.332 & 0.58 & 32 & Good (residual deformity $8^{\circ}$ ) \\
\hline 6 & 0.396 & 0.52 & 29 & Excellent \\
\hline 7 & 0.216 & 0.64 & 40 & Good (limb shortening $2.6 \mathrm{~cm}$ ) \\
\hline 8 & 0.000 & 0.74 & $32 *$ & Excellent \\
\hline 9 & 0.493 & 0.49 & 26 & Excellent \\
\hline 10 & 0.453 & 0.51 & 30 & Excellent \\
\hline 11 & 0.000 & 0.77 & $30^{*}$ & Excellent \\
\hline 12 & 0.233 & 0.56 & 33 & Good (limb shortening $3 \mathrm{~cm}$ ) \\
\hline 13 & 0.680 & 0.57 & 23 & Excellent \\
\hline 14 & 0.276 & 0.49 & 36 & Excellent \\
\hline 15 & 0.428 & 0.58 & 32 & Excellent \\
\hline 16 & 0.103 & 0.63 & 33 & Excellent \\
\hline
\end{tabular}

* For patients without hematoma, the time of bone healing was calculated from the time after the operation.

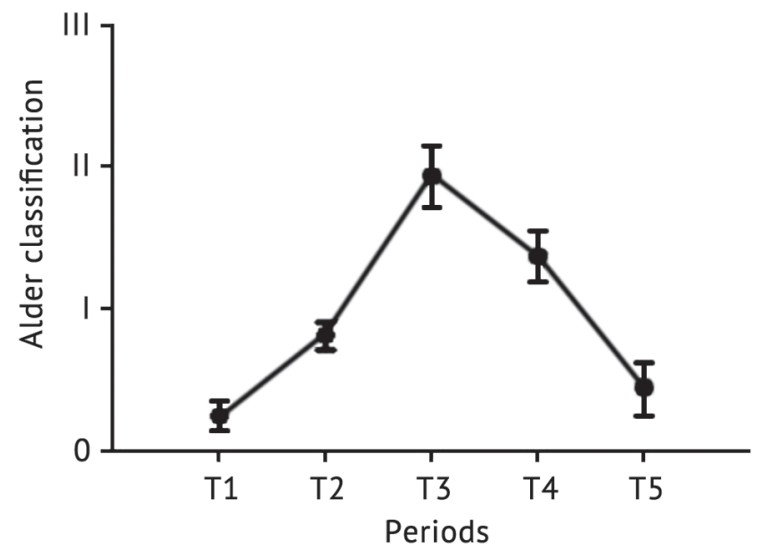

Fig. 7 The picture shows the trend of Adler's blood flow rating in different stages of accordion operation

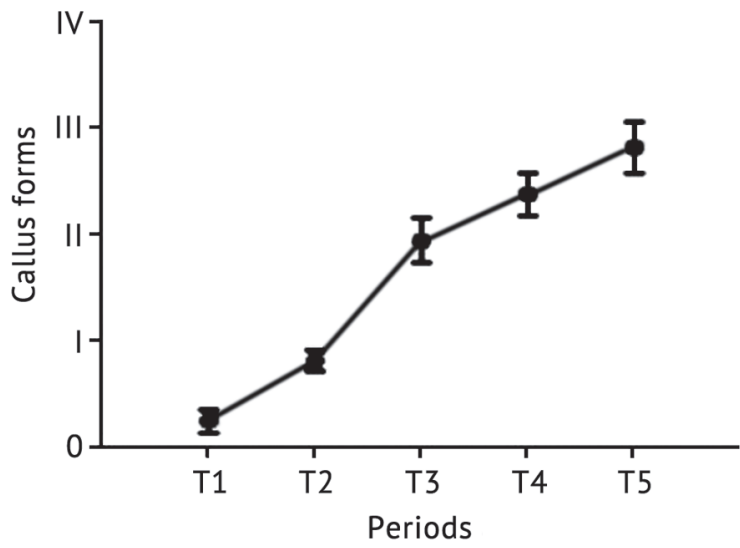

Fig. 8 The picture shows the trend of the degree of callus mineralization at the docking site in each stage of accordion operation

\section{DISCUSSION}

Bone transport technology is to transform mechanical signals of tensile stress into biological signals $[1,2]$ to promote the regeneration of bone, blood vessels and soft tissue. It provides the possibility to cure defect, infection, deformity and other diseases of bone or soft tissue that are difficult to treat clinically. At present, it has been widely used clinically for the treatment of bone defects caused by open fractures of the extremities $[4,6]$ and chronic osteomyelitis. However, many complications of it have been gradually found in the treatment, which affects the treatment time. With the wide application of this technique in clinical practice, the incidence of complications increases gradually. The difficulty 
of union of the docking site is one of them, which determines the length of the treatment period of bone migration. Accordion technology can promote bone healing on the docking site and solve a difficult problem in bone transport technology. It is a method to promote bone healing utilizing alternating compression-distraction stress stimulation. The possible mechanism is that compression stress changes the micro-environment at the docking site [21], which fully activates the bone healing system, and tensile stress promotes microcirculation regeneration at the docking site [22], amplifies the compression effect, and accelerates bone healing. However, there are significant differences in the effectiveness of accordion technique in the present literature. Giotakis et al. [8] reported that accordion technology was used to treat five cases of nonunion at the docking site, and two cases were successful. Hatzokos et al. [23] reported eight cases were treated with it, and six cases achieved bone healing. This is because the operation stage of accordion is in the early stage of bone healing, $\mathrm{X}$-ray cannot effectively monitor the formation of callus [24], and there is a lack of timely and effective feedback on the specific operation plan of accordion, so clinicians can only formulate the plan according to experience, which ultimately leads to the difference in its effectiveness. Therefore, objective and effective monitoring indicators are needed to feed back the formation of callus during accordion operation.

High-frequency color doppler ultrasound can not only detect the formation of callus in the process of bone healing earlier than X-ray, but also observe the changes of hematoma, blood flow signal and surrounding soft tissue around the bone fracture end, which is the unique advantage of ultrasound. A. Poposka et al. [19] used ultrasound to observe the formation of new bone in the traction osteogenic region during bone transport, and found that the formation of new callus could be detected after two weeks of distraction. Su HQ et al. [14] used ultrasound to observe the bone healing process of humeral fracture, and found that blood flow signal could be found at the broken end of bone three days after the fracture, and reached the peak 3-4 weeks after the fracture, and the trend of bone healing could be predicted according to the richness of it. In this experiment, ultrasound found that there was no hematoma when the docking site was contacted, while 14 of the 16 patients developed hematoma when it was compressed. The possible reason is that when the docking site is compressed, microfracture, capillary rupture and soft tissue necrosis occur, resulting in hematoma, at which the microenvironment is similar to that of fresh fracture. The hematoma formed at the docking site could cause local aseptic inflammation, which releases various inflammatory mediators and osteocytokines to collect bone progenitor cells that can be turned into osteoblasts and chondrocytes, so as to initiate bone healing [21]. In all the 14 patients with hematoma, bone healing occurred at the docking site (the average time of bone healing was $30.8 \pm 4.9$ weeks), and it was found that the size of the hematoma was linearly correlated with the bone healing time. The bigger the hematoma, the shorter the bone healing time. Therefore, ultrasound can not only determine the direction of bone healing according to the richness of blood flow, but also predict the time of bone healing earlier according to the hematoma range formed. However, among the 16 patients in this study, 14 had hematoma and 2 had no hematoma, so the incidence of hematoma was $87.5 \%$. For patients without hematoma, ultrasound cannot predict the specific time of bone healing, so this method has certain limitations. Further studies and expanded sample size are needed to determine the effectiveness of this method, so as to further guide clinical practice.

For the two patients without hematoma, ultrasound found that the blood supply and the degree of bone callus mineralization at the docking site during accordion operation were significantly lower than those in the patients with hematoma. It may be that the blood supply to the docking site and surrounding tissues of the patient was seriously damaged, and there was no obvious blood supply, resulting in slow growth of callus and bone healing difficulties. Ilizarov [25] blocked the blood supply of muscle tissue, intramedullary and periosteal membrane in the model of bone regeneration, and found no new bone formation. If one of them was blocked, the rate of new bone formation was slower than normal. It can be seen that for patients without hematoma, although ultrasonic monitoring can not predict the specific time of bone healing, it can determine the trend of bone healing, which has certain guiding significance for clinical practice. In order to avoid the occurrence of bone nonunion at the docking site, the research group changed to the use of bone cortical exfoliation. After the operation, hematoma was found at the docking site, and the accordion operation was continued. The blood supply and the degree of bone callus mineralization was significantly improved compared with that before the operation. Finally, the bone healed at the docking site, and the healing time was similar to that of the patients with hematoma. 
Therefore, hematoma played an obvious role in promoting bone healing, and other studies had shown that after the removal of hematoma at the fracture end, delayed fracture healing and nonunion could be caused $[26,27]$. This research group further considers that the formation of hematoma may be a necessary condition for accordion operation, which needs to be further confirmed.

Ultrasound observation revealed hematoma in patients was gradually organized from the periphery to the center in the intermittent period. The echo intensity of it gradually changed from anechoic or hypoechoic to isoechoic, blood flow signal began to appear around the hematoma, and bone callus gradually formed. In the distraction stage, blood flow signal increased significantly, and the larger the hematoma, the lower the RI mean at two weeks of compression. Su HQ et al. [14] found that the richer the blood flow, the lower the RI average. This indicates that hematoma can induce vascular reaction and improve blood supply around the docking site in the process of bone healing, so as to promote bone healing.

Ultrasound was used to continuously monitor the changes of blood flow signal and new callus at each stage of accordion operation. It was found that before the retraction, the Adler grade of blood flow at the docking site gradually increased, and gradually decreased after the retraction. However, the mineralization degree of new callus continued to increase. It can be seen that in the process of bone healing promoted by accordion technology, the growth of callus not only depends on blood supply, but also can be promote by the compression stress. The possible reason is that the compression stress leads to the collapse of the vascular cavity and the decrease of blood flow signal, resulting in local tissue hypoxia. This environment induces the production of hypoxia-inducible factor (HIF-1), which mediates the expression of vascular endothelial growth factor (VEGF) and promotes the formation of callus [28]. In addition, compressive stress can maintain high expression of multiple osteogenic factors generated during distraction [29], further promoting callus growth. Therefore, accordion technology can improve the microenvironment at the docking site and promote bone healing by applying compression stress.

When the docking site was in contact again, ultrasonic observation showed that the contact between the two sides of it was less compact. If continue to compress it for one week, it could not only prevent the absorption of new bone callus, but also keep it under pressure for a long time, which was conducive to the formation of bone connection.

\section{CONCLUSION}

To sum up, (1) as an effective monitoring method in the early stage of bone healing, high-frequency color Doppler ultrasound can dynamically monitor the change trend of hematoma, blood flow and new callus at the docking site during accordion operation, so as to predict the tendency of bone healing at the docking site and reduce the occurrence of non-bone healing.
(2) This study can preliminarily demonstrate that the compression stress applied in accordion technique can promote the bone healing at the docking site, which can provide reference for the further mechanism research of it. However, due to the small number of cases collected in this study, it is still necessary to further expand the sample size to verify the above viewpoints.

\section{REFERENCES}

1. Grubor P., Mitković M., Grubor M., Mitković M., Meccariello L., Falzarano G. Biomechanical Stability of Juvidur and Bone Models on Osteosyntesic Materials. Acta Inform. Med., 2016, vol. 24, no. 4, pp. 261-265. DOI: 10.5455/aim.2016.24.261-265

2. Bisaccia M., Rinonapoli G., Meccariello L., Caraffa A., Cukierman B., Iborra J.R. The Challenges of Monoaxial Bone Transport in Orthopedics and Traumatology. Ortop Traumatol. Rehabil., 2017, vol. 19, no. 4, pp. 373-378. DOI: 10.5604/01.3001.0010.4646

3. Khan M.S., Di Giacomo L.M., Bisaccia M., Azzam W., Jatoi A., Rollo G., Meccariello L. Ilizarov technique, satisfactory outcome with limited resources. Clin. Cases Min. Bone Metab., 2018, vol. 15, no. 2, pp. 221-226.

4. Grubor P., Milicevic S., Grubor M., Meccariello L. Treatment of Bone Defects in War Wounds: Retrospective Study. Med Arh., 2015, vol. 69, no. 4, pp. 260-264. DOI: 10.5455/medarh.2015.69.260-264

5. Aktuglu K., Erol K., Vahabi A. Ilizarov bone transport and treatment of critical-sized tibial bone defects: a narrative review. J. Orthop. Traumatol., 2019, vol. 20, no. 1, pp. 22. DOI: 10.1186/s10195-019-0527-1

6. Grubor P., Falzarano G., Grubor M., Piscopo A., Franzese R., Meccariello L. Treatment of the chronic war tibial osteomyelitis, Gustilo type IIIB and Cierny-Mader IIIB, using various methods. A retrospective study. EMBJ EuroMediterranean Biomedical Journal, 2014, vol. 9, no. 2, pp. 7-18. DOI:10.3269/1970-5492.2014.9.2

7. Robinson P.M., Papanna M., Younis F., Khan S.A. Arthroscopic debridement of docking site in Ilizarov bone transport. Ann. R. Coll. Surg. Engl., 2010, vol. 92, no. 5, pp. 437-438. DOI: 10.1308/003588410x12699663903719b

8. Giotakis N., Narayan B., Nayagam S. Distraction osteogenesis and nonunion of the docking site: is there an ideal treatment option? Injury, 2007, vol. 38, no. Suppl 1, pp. S100-S107. DOI: 10.1016/j.injury.2007.02.015

9. Moghaddam M.H., Vahedi E. Effect of decortications on union rate of tibial plating. Orthopedics, 2015, vol. 38, no. 3, pp. e213-e216. DOI: 10.3928/01477447-20150305-59 
10. Tall M., Bonkoungou D., Sawadogo M., Da S.C., Toe M.F.; Bone and Joint Trauma Study Group (GETRAUM). Treatment of nonunion in neglected long bone shaft fractures by osteoperiosteal decortication. Orthop. Traumatol. Surg. Res., 2014, vol. 100, no. 6 Suppl., pp. S299-S303. DOI: 10.1016/j.otsr.2014.07.005

11. Manni M., Bisaccia M., Rinonapoli G., Schiavone A., Meccariello L., Mccabe S.J., Bisaccia O., Vicente C.I., Cappiello A., Caraffa A. Reliability, Feasibility and Value of Ecography in Clinical-functional Results in Patients Affected by Carpal Tunnel Syndrome: is There a Correlation? Acta Inform. Med., 2017, vol. 25, no. 1, pp. 44-48. DOI: 10.5455/aim.2017.25.44-48

12. Bisaccia M., Rinonapoli G., Meccariello L., Bisaccia O., Ceccarini G., Rollo C., Ibáñez-Vicente C., Cervera-Irimia J., Sánchez-Sánchez F., RibesIborra A., Gomez-Garrido D., Caraffa A. Validity and Reliability of Mini-Invasive Surgery Assisted by Ultrasound in Achilles Tendon Rupture. Acta Inform. Med., 2019, vol. 27, no. 1, pp. 40-44. DOI: 10.5455/aim.2019.27.40-44

13. Bisaccia M., Caraffa A., Meccariello L., Ripani U., Bisaccia O., Gomez-Garrido D., Carrado-Gomez M., Pace V., Rollo G., Giaracuni M., Rinonapoli G. Displaced patella fractures: percutaneous cerclage wiring and second arthroscopic look. Clin. Cases Miner. Bone Metab., 2019, vol. 16, pp. 48-52.

14. Su H.Q., Zhuang X.Q., Bai Y., Ye H.H., Huang X.H., Lu B.B., Lu S.L., Nong D.Y., Wang W. Value of ultrasonography for observation of early healing of humeral shaft fractures. J. Med. Ultrason., 2013, vol. 40, no. 3, pp. 231-236. DOI: 10.1007/s10396-012-0426-Z

15. Chachan S., Tudu B., Sahu B. Ultrasound monitoring of fracture healing: is this the end of radiography in fracture follow-ups? J. Orthop. Trauma, 2015, vol. 29, no. 3, pp. e133-e138. DOI: 10.1097/BOT.0000000000000207

16. Andrade N., Aggrawal N., Jadhav G., Sahu V., Mathai P.C. To determine the efficacy of ultrasonography in the evaluation of bone fill at the regenerate site for mandibular distraction osteogenesis over clinical and radiographic assessment. An in vivo prospective study. J. Oral Biol. Craniofac. Res., 2018, vol. 8, no. 2, pp. 89-93. DOI: 10.1016/j.jobcr.2018.04.005

17. Richter D., Hahn M.P., Ostermann P.A., Ekkernkamp A., Muhr G. [Ultrasound follow-up of callus distraction - an alternative to roentgen diagnosis?]. Langenbecks Arch. Chir. Suppl. Kongressbd., 1996, vol. 113, pp. 931-933. (in German)

18. Adler D.D., Carson P.L., Rubin J.M., Quinn-Reid D. Doppler ultrasound color flow imaging in the study of breast cancer: preliminary findings. Ultrasound Med. Biol., 1990, vol. 16, no. 6, pp. 553-559. DOI: 10.1016/0301-5629(90)90020-d

19. Poposka A., Atanasov N., Dzoleva-Tolevska R. Use of ultrasonography in evaluation of new bone formation in patients treated by the method of Ilizarov. Prilozi, 2012, vol. 33, no. 1, pp. 199-208.

20. Paley D., Catagni M.A., Argnani F., Villa A., Benedetti G.B., Cattaneo R. Ilizarov treatment of tibial nonunions with bone loss. Clin. Orthop. Relat. Res., 1989, no. 241, pp. 146-165.

21. Barker K.L., Lamb S.E., Simpson A.H. Functional recovery in patients with nonunion treated with the Ilizarov technique. J. Bone Joint Surg. Br., 2004, vol. 86, no. 1, pp. 81-85.

22. Bouletreau P.J., Warren S.M., Spector J.A., Steinbrech D.S., Mehrara B.J., Longaker M.T. Factors in the fracture microenvironment induce primary osteoblast angiogenic cytokine production. Plast. Reconstr. Surg., 2002, vol. 110, no. 1, pp. 139-148. DOI: 10.1097/00006534-200207000-00025

23. Hatzokos I., Stavridis S.I., Iosifidou E., Karataglis D., Christodoulou A. Autologous bone marrow grafting combined with demineralized bone matrix improves consolidation of docking site after distraction osteogenesis. J. Bone Joint Surg. Am., 2011, vol. 93, no. 7, pp. 671-678. DOI: 10.2106/JBJS.J.00514

24. Caruso G., Lagalla R., Derchi L., Iovane A., Sanfilippo A. Monitoring of fracture calluses with color Doppler sonography. J. Clin. Ultrasound., 2000, vol. 28, no. 1, pp. 20-27. DOI: 10.1002/(sici)1097-0096(200001)28:1<20::aid-jcu3>3.0.co;2-w

25. Ilizarov G.A. The tension-stress effect on the genesis and growth of tissues. Part I. The influence of stability of fixation and soft-tissue preservation. Clin. Orthop. Relat. Res., 1989, no. 238, pp. 249-281.

26. Echeverri L.F., Herrero M.A., Lopez J.M., Oleaga G. Early stages of bone fracture healing: formation of a fibrin-collagen scaffold in the fracture hematoma. Bull. Math. Biol., 2015, vol. 77, no. 1, pp. 156-183. DOI: 10.1007/s11538-014-0055-3

27. Ozaki A., Tsunoda M., Kinoshita S., Saura R. Role of fracture hematoma and periosteum during fracture healing in rats: interaction of fracture hematoma and the periosteum in the initial step of the healing process. J. Orthop. Sci., 2000, vol. 5, no. 1, pp. 64-70. DOI: 10.1007/s007760050010

28. Mori S., Akagi M., Kikuyama A., Yasuda Y., Hamanishi C. Axial shortening during distraction osteogenesis leads to enhanced bone formation in a rabbit model through the HIF-1alpha/vascular endothelial growth factor system. J. Orthop. Res., 2006, vol. 24, no. 4, pp. 653-663. DOI: 10.1002/ jor.20076

29. Alzahrani M.M., Anam E.A., Makhdom A.M., Villemure I., Hamdy R.C. The effect of altering the mechanical loading environment on the expression of bone regenerating molecules in cases of distraction osteogenesis. Front. Endocrinol. (Lausanne), 2014, vol. 5, p. 214. DOI: 10.3389/ fendo.2014.00214

Received: 08.04.2021

\section{Information about the authors:}

1. Guoyu He,

The second Hospital of Shanxi Medical University, Taiyuan, China

2. Xin Zhou,

The second Hospital of Shanxi Medical University, Taiyuan, China 3. Ying Shi,

The second Hospital of Shanxi Medical University, Taiyuan, China

4. Dong Wang,

The second Hospital of Shanxi Medical University, Taiyuan, China

5. Jing Yu,

The second Hospital of Shanxi Medical University, Taiyuan, China 6. Hao Xing,

The second Hospital of Shanxi Medical University, Taiyuan, China
7. Haiyang Yin,

The second Hospital of Shanxi Medical University, Taiyuan, China

8. Jin Zhang,

The second Hospital of Shanxi Medical University, Taiyuan, China 9. Hong Liu,

The second Hospital of Shanxi Medical University, Taiyuan, China 10.Baona Wang,

The second Hospital of Shanxi Medical University, Taiyuan, China 11.Yingwei Jia,

The second Hospital of Shanxi Medical University, Taiyuan, China 12. Yonghong Zhang,

The second Hospital of Shanxi Medical University, Taiyuan, China, Email: yhzhy@139.com 